\title{
Niklas der Fisch
}

\section{Bericht über ein Erzähltheaterprojekt mit italienischen \\ Sekundarschülern}

\section{Marina Bertino}

\begin{abstract}
Zusammenfassung
Im folgenden Artikel wird über die wichtigsten Momente eines Theaterprojekts im Rahmen des fremdsprachlichen Deutschunterrichts an einer italienischen Sekundarschule berichtet. Als Textgrundlage diente eine sizilianische Legende. Die Inszenierung der Legende basierte auf den Techniken des Erzähltheaters (Teatro di narrazione) im Sinne des Sozialtheaters, einem wichtigen Genre im Neuen Theater (Teatro nuovo) Italiens. Dieser Beitrag beleuchtet, wie bereits im Anfängerunterricht das Erzählen und Inszenieren eigener Geschichten, Märchen und Mythen in der Fremdsprache eine motivierende und lernfördernde Rolle spielen kann.
\end{abstract}

\section{Einleitung}

Auf der Internationalen Deutschlehrertagung 2005 in Graz zeigte ein indischer Kollege in der Sektion „Dramapädagogik und experimentelle Lehrformen“ ein mit seinen Schülern gedrehtes Video. Im Stile eines Bollywood-Musicals stellten die Schüler ein indisches Versepos sehr eindrucksvoll auf Deutsch dar. Dabei wurde die Fremdsprache als Instrument der Bewusstmachung und Vermittlung der eigenen kulturellen Wurzeln und als Begegnungsplatz der verschiedenen Kulturen verwendet, das heißt: es fand eine direkte Übertragung der indischen Denkweise und der damit verbundenen Gestik in eine andere Sprache statt.

Diese Didaktisierung des indischen Video-Dramas lieferte die erste Anregung, aus der sizilianischen Legende über den Fischmenschen Colapesce ein Theaterstück mit meinen Schülern (DaF-Anfänger, europäisches Referenzrahmenniveau A1-A2) zu erarbeiten, um im darstellerischen Prozess soziale, kognitive und emotionale Aspekte zu betonen.

Die Gestalt des Fischmenschen lässt sich in den Märchen oder Legenden von vielen Kulturen finden. Ebenso ist das Thema der Verwandlung in mythologischen Geschichten sehr verbreitet.

Die Idee, Geschichten aus dem eigenen Kulturkreis in einer Fremdsprache zu inszenieren, habe ich dann in einem Gespräch mit potentiellen Betreuern einer 
Promotion zu diesem Thema im Sommer 2006 am University College Cork Ireland (UCC) diskutiert, woraus die Idee für ein konkretes dramapädagogisches Schulprojekt erwuchs, in dem sich mit folgenden Fragen auseinander gesetzt werden sollte:

- Wie ist das Verhältnis zwischen dem szenischen Erzählen von eigenen, auf Märchen, Mythen und Legenden, aber auch Tagesereignissen basierenden Geschichten und der Aneignung sprachlicher und darstellerischer Kompetenz in der Fremdsprache?

- Wie lässt sich mittels der Techniken des Erzähltheaters (Teatro di narrazione) eine sizilianische Erzählung in einen deutschsprachigen dramatischen Text umwandeln?

Diese Fragen waren der Ausgangspunkt, um mit den Schülern ein Skript zu erarbeiten und dann am vom Goethe-Institut Turin veranstalteten Wettbewerb Mit Deutsch auf die Bühne! teilzunehmen. Im Folgenden werde ich über das Projekt mit meinen Schülern berichten und einen Exkurs über die Tradition des sozial engagierten Erzähltheaters in Italien und Sizilien, das für das Projekt grundlegend ist, voran stellen.

\section{Das Erzähltheater in Italien und in Sizilien}

Erzähltheater gehört ursprünglich zu den Vorformen des Theaters und zur Kunst der Dithyramben und Rhapsoden der Antike, die von den Bänkel- und Moritatensängern des Mittelalters bis in die Neuzeit gepflegt wurde und auch mit dem weltweit berühmten Mistero Buffo (1969) von Dario Fo assoziiert wird.

Die Form des „Teatro di narrazione“ (Erzähltheaters) hat sich in Italien seit den 80er Jahren rasch entwickelt. Hauptvertreter dieses neuen Genres sind Marco Baliani, Marco Paolini, Mario Perrotta und die Sizilianer Davide Enia und Vincenzo Pirrotta. Sie sind oft die Verfasser und zugleich die Darsteller ihrer Stücke. Es handelt sich um einen Theaterstil, bei dem der Hauptteil des Skripts von einem Narratore (Erzähler) bzw. Narra-attore (Erzähl-Darsteller) erzählt und dargestellt wird. Manchmal haben zusätzliche Schauspieler die Aufgabe, das Erzählte weiterzuspielen. Meistens ist aber ein einziger Darsteller auf der Bühne, der die Geschichte „erzählt“. Dieses Theatergenre entstand mit dem schon erwähnten Mistero Buffo von Dario Fo. Der Erzähler führt in der jeweiligen Szene die Handlungen aus und spielt dabei verschiedene Figuren. In Sizilien kommt dieses Genre ursprünglich aus den traditionellen Formen des Cuntu ('Erzählen' in sizilianischer Mundart) und der Opera dei Pupi (Sizilianisches Marionettentheater), die bis zum letzten Jahrhundert erhalten geblieben sind. In dieser Traditionslinie wird heute das Erzähltheater von Vincenzo Pirrotta weitergeführt, der als Poeta del Cunto (Dichter des Erzählens) bekannt ist. Seine Hauptlehrer sind die Brüder Cuticchio aus Palermo, die berühmtesten Figurentheaterdarsteller in Sizilien. Mit seiner Tragödie Euminidi (2004) klagt 
Pirrotta, in Anlehnung an einen griechischen Mythos, die Korruption des heutigen italienischen Justizsystems an. Teilweise erzählt dieses Stück die tragische Handlung, teilweise vermittelt es sie in Form von Liedern. Es ist deswegen ein gutes Beispiel für einen besonderen Stil des Erzähltheaters.

$\mathrm{Zu}$ betonen ist, dass sich das Erzähltheater in Italien oft als Sozialtheater (Teatro civile) versteht, egal ob seine Geschichten aus der Antike (Euminidi von Pirrotta), aus dem Mittelalter (Mistero Buffo von Fo) oder aus der neuen Literatur (Kohlhaas, Balianis freie Adaption von Kleists Michael Kohlhaas, 1990) kommen, vor allem um die negativen Seiten Italiens zu erforschen und sie durch die Performance zu offenbaren. So handelt Balianis Corpo di stato (1998) von Aldo Moros Mördern aus den Reihen der Roten Brigaden im Jahr 1978; in Olivetti (Regie Gabriele Vacis, 1996) erzählt Laura Curino von Camillo und Adriano Olivetti, den Begründern des Olivetti Business Empires; in Il racconto del Vajont (1995) erläutert Marco Paolini die Tragödie des Vajont-Dammbruchs im Jahr 1963 mit mehr als tausend Opfern. In Maggio '43 (2004) von Davide Enia wird von der alliierten Invasion und dem Bombenangriff auf Palermo während des Zweiten Weltkriegs berichtet. In Radio Clandestina (2000) und in Scemo di guerra (2004) erzählt Ascanio Celestini von der Besetzung Roms durch die Nazis und von der folgenden Befreiung durch die Amerikaner.

In der Theatergruppe haben wir, wie im Weiteren berichtet wird, mit den Darstellungstechniken des sozialen Erzähltheaters experimentiert.

\section{DaF in Italien und der Wettbewerb: Mit Deutsch auf die Bühne!}

Deutsch wird in Italien meist ab dem 15. Lebensjahr als zweite oder weitere Fremdsprache gelernt. In Süd-Italien wird es vor allem als dritte Fremdsprache gewählt. Das Spanische ist allerdings seit ein paar Jahren die von den Schülern bevorzugte Sprache, auch dank der starken Ähnlichkeiten mit dem Italienischen. Das bedeutet in den Klassen mit Deutschunterricht immer weniger Schüler, kleinere Gruppen, die manchmal nur aus 8-12 Schülern bestehen und aus einer gesamten Klasse herausgenommen werden. Die Arbeitsbedingungen für viele Deutschlehrkräfte sind schwierig; sie werden Jahr für Jahr aus rein bürokratischen Gründen von einer Schule zu anderen versetzt. Der damit einher gehende Mangel an Kontinuität wirkt sich negativ auf die Qualität des Unterrichts aus.

In dieser schwierigen Situation versuchen die Goethe-Institute, GoetheZentren und ACIT (Associazione Culturale Italo-Tedesca), die Arbeit an der deutschen Sprache mit verschiedenen Initiativen und Veranstaltungen $\mathrm{zu}$ unterstützen.

Eine dieser Initiativen ist der Wettbewerb Mit Deutsch auf die Bühne!, der in Italien jedes Jahr vom Goethe Institut Turin innerhalb des europäischen Jugendtheaterfestivals Lingue in Scena! Langues en Scène! Sprachen in Szene! organisiert wird. 
Schulklassen aus ganz Italien mit Deutschlernern im Alter von 15-19 Jahren können am Wettbewerb in Gruppen von zirka 10 Personen mit einem Theaterstück von 30 Minuten teilnehmen. Erzieherische und didaktische Ziele der Initiative sind u. a. die Entwicklung sprachlicher und darstellerischer Kompetenzen sowie die kritische Auseinandersetzung mit der Komplexität der Theaterwelt aus der Darsteller- bzw. Publikumsperspektive. Die aus Vertretern des Goethe-Instituts, der Universität Turin, des professionellen Theaters und des Stadtrats Turin zusammengesetzte Wettbewerbskommission wählt das beste Stück nach folgenden Kriterien: Kreativität, Originalität, Sprachkompetenz, darstellerische Kompetenz, Regie, Bühnenbild und Inszenierung.

Voraussetzung zur Teilnahme am VII. nationalen Wettbewerb Mit Deutsch auf die Bühne war der dreitägige Workshop für Deutschlehrkräfte Theatermethoden im DaF-Unterricht, der im Oktober 2006 vom Goethe-Institut Turin veranstaltet wurde. Diese Intensiv-Veranstaltung unter Leitung der Theaterpädagoginnen Alice Herberger und Sigrid Unterstab war äußerst ergiebig. Ich persönlich hatte so auch die Gelegenheit, auf Deutsch meine langjährige Theaterausbildung (in Workshops nach den Ansätzen von Grotowski, Boal, Shintaido, Living Theatre) aufzufrischen, aber vor allem bekam ich den Ansporn, ein Theaterprojekt über die Legende von Colapesce mit meinen Schülern zu realisieren.

Die Theatergruppe aus der Sekundarschule für Tourismus „IISS De Nicola“ in Catania bestand aus 13 Teilnehmern (DaF-Anfänger, Niveau A1-A2), und zwar 7 Schülerinnen und 3 Schülern als Schauspielern und 3 Schülerinnen als Bühnenbildnerinnen. Die Teilnehmer lernten vormittags vier Stunden Deutsch und nahmen nachmittags, vom November 2006 bis Mai 2007, ein- oder zweimal pro Woche, freiwillig insgesamt 80 Stunden lang an der Theaterwerkstatt teil. Regieassistentin war Magda Brudziak aus Berlin, die mit der Gruppe nur auf Deutsch sprach und das Projekt, trotz teilweise schlechter schulischer Rahmenbedingungen (Räume, Zeit, finanzielle Mittel u.a.), mit mir zusammen realisierte.

Für die Teilnahme am Wettbewerb Mit Deutsch auf die Bühne 2007 wurden dann sieben Schultheaterprojekte aus ganz Italien ausgewählt. Als am 26. März 2007 die ausgewählten Schuldelegationen ihr Stück in Turin aufführten, ging unsere Gruppe aus Catania mit dem Theaterstück Niklas der Fisch, breve opera subacquea aus dem Wettbewerb als Gewinner hervor.

Als Preis durfte unsere Theatergruppe vom 14. bis 18. Mai 2007 beim Festival Sprachen in Szene! Italien mit der Aufführung unseres Stückes vertreten und auch an einem besonderen Workshop zur Commedia dell'Arte teilnehmen.

Das war für uns eine große Anerkennung des Experiments, eine Legende aus unserer Kultur in einer Fremdsprache zu inszenieren. Die wichtigsten Momente dieses langen gemeinsamen künstlerisch-kreativen Arbeitsprozesses, der zu diesem Ergebnis führte, möchte ich im weiteren Verlauf darstellen. 


\section{Das Experiment mit dem Stück Niklas der Fisch}

Im Sinne einer produktiven Arbeit unserer Theatergruppe war es von Anfang an wichtig, von den Teilnehmern ein hohes Maß an Selbstverantwortung zu verlangen und ihnen Mitbestimmungsrecht einzuräumen. Eine Aufführung ist ein Gemeinschaftsprodukt und kann nur gelingen, wenn alle selbständig und verantwortungsbewusst mitarbeiten.

Zur Förderung der Zusammenarbeit haben wir uns von Marco Baliani inspirieren lassen. Er erzählt in seinem Buch Pinocchio Nero, wie seine Theatergruppe kooperativ und mit dem gleichen Engagement wie eine Fußballmannschaft arbeitet und spielt:

Come nel gioco del calcio si è squadra solo quando si riesce a sentire che ognuno è al servizio degli altri, che senza l'altro non ce la puoi fare, l'opposto del teatro che insegnano nelle nostre accademie e scuole dove si punta tutto sull'interpretazione individuale, sull'accanimento a emergere, a sgomitare contro l'altro pur di eccellere e farsi vedere. (Baliani 2005: 60) ${ }^{1}$

Unsere Projektarbeit bzw. Theaterwerkstatt, die in die Teilnahme am nationalen Wettbewerb Mit Deutsch auf die Bühne mündete, vollzog sich in sieben Phasen:

- Reflexion über den Text Colapesce (Textverstehen);

- Generierung von Inszenierungsideen und dramaturgische Textbearbeitung;

- Erarbeitung des Erzähltheaterstücks auf Deutsch;

- Entwicklung des freien Körper- und Stimmausdrucks (Auflockerungsphase);

- Arbeit an der Darstellungs- und Erzählfähigkeit auf Deutsch (Sprech- und Gesangsübungen);

- Aufführung des Theaterstücks;

- Erstellung einerVideo-Adaption desErzähltheaterstücks. (EinVideo-clip kann hier herunter geladen werden)

\footnotetext{
${ }^{1}$ Wie im Fußball, ist man erst eine Mannschaft, wenn man spüren kann, dass der eine für den anderen spielt, dass man es ohne den anderen nicht schaffen kann. Das ist das Gegenteil des Theaters, das uns (in Italien) in den Schauspielschulen beigebracht wird, wo alles auf individuelle Interpretation gesetzt wird, auf die Gier herauszukommen, und wo mit Ellenbogen gegen andere gekämpft wird, nur um Erfolg zu haben, um aufzufallen. (Übersetzung MB)
} 


\subsection{Von der Legende Colapesce zum Erzähltheaterstück}

Es gibt mehr als zehn Versionen der Legende von Colapesce. Erst haben wir mit der Version aus Messina, die in den Italienischen Märchen (Fiabe Italiane) von Italo Calvino zu finden ist, gearbeitet. Hier einige Auszüge, die für unser Projekt wichtig geworden sind:

Es war einmal in Messina eine Mutter, die hatte einen Sohn, der hieß Cola. Vom frühen Morgen bis zum Abend blieb er immer im Meer und badete. Die Mutter rief ihm oft vom Ufer aus zu: Cola, Cola, komme an Land! Was machst du? Du bist doch nicht etwa ein Fisch!Ëines Tages [...] als sie sah, dass sie ihn mit Rufen nicht mehr erreichen könne, schickte ihm einen wilden Fluch nach: Cola, dass du doch ein Fisch würdest!"[...] der Fluch der Mutter erfüllte sich sofort: Cola wurde halb Mann, halb Fisch. [...] Das Gerücht, dass im Meer von Messina ein Wesen, halb Mensch, halb Fisch, sei, gelangte bis zum König. Der König befahl allen Schiffen, wenn einer von ihnen Cola Pesce sähe, so möge er ihm sagen, der König wolle ihn sehen. [...] Der König empfing ihn huldvoll. Cola Pesce", sprach er, "da du ein so guter Schwimmer bist, sollst du mir um ganz Sizilien herumschwimmen und mir kundtun, wo das Meer am tiefsten ist und was man dort sieht!"[...] Cola Pesce erzählte, er habe auf dem Meeresgrund Berge, Täler, Höhlen und Fische aller Art gesehen, aber er habe nur Furcht gehabt, als er das Cap Faro umschwommen habe, denn dort sei es ihm nicht gelungen, den Meeresgrund zu finden. [...] Der König konnte seine Neugier nicht mehr bezähmen und sprach: SStürze dich vom Turm des Felsens auf Cap Faro ins Meer!"[...] Cola Pesce stürzte sich von dort oben kopfüber hinunter. [...] Endlich tauchte er auf, aber er war totenbleich. [...] Ïch habe einen riesigen Fisch gesehen mit einem so großen Maul, dass ein ganzes Schiff darin Platz hätte. [...]"Der König hörte mit offenem Munde zu; aber seine verfluchte Neugier, wie tief das Meer dort sei, ließ ihn nicht ruhen. [...] Da nahm der König seine Krone vom Haupt [...]. Mit einem großen Schwung warf er sie ins Meer. "Geh und bring sie mir zurück, Cola!"befahl er. [...] "Wenn es Euer Wille ist, Majestät, dann werde ich eben hinabsteigen, aber [...] lasst mir eine Handvoll Linsen geben. Wenn ich dem Ungeheuer entrinne, dann werde ich wiederkehren, wenn Ihr dagegen die Linsen aufsteigen seht, dann nehmt das als Zeichen, dass ich nie wieder auftauchen werde."[...] Cola stieg in die Tiefe. Der König aber wartete und wartete, und endlich, nach langem vergeblichem Warten, erschienen die Linsen auf der Oberfläche des Wassers. Auf die Rückkehr von Cola Pesce aber wird heute noch gewartet. (Calvino 1993: 151)

Diese Version von Calvino haben wir mit derjenigen aus Catania vermischt, in der die ganze Geschichte am sizilianischen Hof des Stauferkaisers Friederich II. angesiedelt ist, so wie sie noch in anderen Versionen erzählt wird.

Bei Darstellungen von Geschichten kann beispielsweise ein Zwischenerzähler eingefügt werden und man kann aus der aristotelischen Einheit von Raum und Zeit austreten, um Vergangenes im Jetzt erfahrbar zu machen (vgl. Baliani 2005). Gleich zu Beginn des Projekts habe ich den Schülern erklärt, warum ich szenisches Spiel in der Fremdsprache in der Form des Erzähltheaters 
vorgeschlagen habe. Erst wird im Stück erzählt, dann wird dargestellt, um in der Fremdsprache durch verschiedene Wahrnehmungssinne nicht nur die Zuschauer bzw. Zuhörer einzubeziehen, sondern auch vor allem die Schüler als Schauspieler aktiv zu beteiligen. Wenn man in einer Fremdsprache ein Märchen bzw. eine Legende interpretiert oder in Szene setzt, kann man sich freier fühlen und einfachere Sprachstrukturen benutzen, als wenn man in der eigenen Sprache interpretieren soll. Die Fremdsprache dient in diesem Falle als Maske, ganz im Sinne der Worte Gilberts in The Critic as Artist von Oscar Wilde:

„Man is least himself when he talks in his own person. Give him a mask, and he will tell you the truth" (Wilde 1913: 185).

Für unsere Gruppe war es eine kreativer Prozess und eine nachhaltig wichtige Erfahrung, den Prosa-Text der sizilianischen Legende in ein Theaterstück umzuformen und dabei eigene Ideen einzubringen. Dabei wurde den Schülern nach und nach die Essenz dieser Legende, einschließlich ihrer archetypischen Merkmale, deutlich.

Unser erstes Treffen begann mit einem gemeinsamen Brainstorming. Im Zentrum der Wandtafel stand der Titel der Legende Cola Pesce. Wir hatten uns nach einer kurzen Diskussion als beste Übertragung des Titels ins Deutsche zunächst für Cola der Taucher, erst einige Monate später für Niklas der Fisch entschieden. Aus freien Assoziationen ergaben sich - zum Teil auch erfundene - Elemente und Personen, die später für den Aufführungstext wichtig wurden: Meer, Schiff, Krone, Strand, Mutter, Kind, König, Königstochter, Hofschiffer.

Schriftlich hat jeder Schüler in Form eines Schemas die Analyse der einzelnen Personen vorgenommen und nach der Erarbeitung dieser verschiedenen schematischen Analysen wurden die Rollen (auch doppelt) verteilt: Niklas, Niklas Gedanken (Pflicht und Recht), seine afrikanische Mutter, die drei Hofnarren Tulpino, Tulpina und Tulpe, der König Friedrich II., die Königstochter Margherita von Sizilien, ein arabischer und ein normannischer Hofschiffer, Hofdamen sowie die Erzählerin, die gleichzeitig eine Taucherin ist. Denn im Theaterstück wird die gesamte Geschichte von dieser Taucherin aus unserer Zeit erzählt:

Eine Taucherin schwimmt im Meer ( = das Publikum im Dunkeln). Sie steigt aus dem Wasser ans Land (= die Bühne). In der Hand hat sie eine Amphore. Aus der Amphore nimmt sie eine Pergamentrolle heraus und liest vor. (Auszug aus dem Text unseres Theaterstücks)

Außerdem haben wir Zitate aus Gedichten und Liedern aus der deutschen Literatur gesucht und gefunden, die mit den Geheimnissen der Tiefe des Meeres zu tun haben und uns beispielsweise von der Sprachästhetik folgender Texte inspirieren lassen: Der Taucher von Friedrich Schiller, Der König im Thule von Johann Wolfgang Goethe. Und zur Verwandlung des Fischmenschen haben wir den sehr bekannten Incipit der Verwandlung von Franz Kafka als Teil unseres Prologs adaptiert:

Als (Gregor Samsa $=$ ) Niklas eines Morgens aus unruhigen Träumen erwachte, fand er sich in seinem Bett zu einem ungeheueren (Ungeziefer 
=) Fischmenschen verwandelt.... Ah nein, Moment, das war doch Kafka...! (aus unserem Theaterstück)

Wenn die Haifische Menschen wären von Bertolt Brechthalf uns, die Geschichte in unsere Tage zu übertragen. Das Projekt hatte nämlich als Sozialhintergrund die Überlegung, die heutige illegale Immigration über das Mittelmeer aufzugreifen. Alle Entscheidungen im Skript zielten auf dieses soziale Problem ab: so ist die Mutter von Niklas Afrikanerin und stellt alle Mütter dar, deren Kinder auf der Flucht nach Europa auf überfüllten Schiffen das Leben riskieren: „Niklaaaas komm an Land!“. Die zwei Hofschiffer, die immer zusammen in den Szenen auftreten und im Chor sprechen, sind im Kontrast ein Araber und ein Normanne, um die Toleranz am sizilianischen Hof des Stauferkaisers Friederich II. anzudeuten. Der Konflikt von Niklas zwischen Pflicht gegenüber der Autorität und Recht auf Leben ist ein ethischer Konflikt wie in der Problematik der illegalen Immigration. Der Epilog unseres Theaterstücks lautet:

Erzählerin: Und heutzutage? Was kann man im Meer Siziliens finden? Farbige Kleider anderer Kulturen, Papiere, und auch wirkliche Kadaver... ... und jeden Tag gibt es viel zu sehen... vor allem sehr schwere Schiffe voll mit Afrikanern auf der Flucht nach der „Festung“ Europa. „Wenn die Haifische Menschen wären... “ Diejenigen, die nie wieder das Land erreichen, sind die neuen Niklas Fische!

Thematische Bilder als Endvorhang, afrikanische Trommeln (Faraualla) (Eine Stimme aus dem Off ) Afrikanische Mutter: Niklaaaas, Komm an Land!

\subsection{Freier Körper- und Stimmausdruck}

Die Aufwärmung der Stimme und des Körpers geschah im Kreis mit dem Ausdruck der fünf Vokale mit Shintaido-Körperbewegungen oder mit einer Auflockerungs- und Aufwärmrunde, in der die Teilnehmer nacheinander Wörter skandierten, so das eine Reihe entstand, zum Beispiel: ja, nein, doch. Das wurde mit einem Klatsch- oder Schnipps-Rhythmus begleitet. Eine Zustimmung (ja): gleiche Richtung und Wiederholung der gleichen Zustimmung ( $j a)$; mit einer Verneinung (nein) ändert sich die Kreisrichtung, mit dem Stopp des Klatschens und mit der Gegenverneinung (doch) wird die vorherige Kreisrichtung wieder aufgenommen. Andere Beispiele: klar, nö, eventuell. Wir haben diese erste Phase der Aufwärmung auch mit Wörtervorschlägen der Gruppe weitergeführt: immer, niemals, manchmal, oder mit den folgenden Farbennamen: rot, schwarz, weiß und dann mit den entsprechenden Gefühlen: Liebe, Hass, Frieden. Es entstand eine hervorragende Gruppendynamik, die Teilnehmer fühlten sich miteinander immer freier, lockerer und auch sprachlich sicherer, dank der Wortspiele und Bewegungen dieser Aufwärmungsphase.

Danach führten wir viel Bewegung im Raum ein: schneller, langsamer, zusätzlich belebt mit Improvisationselementen, die wir für unser späteres Theaterspiel gut gebrauchen konnten: am Strand barfuss wandern, mit einem 
Boot im Meer jemanden suchen, wie Menschen schwimmen, wie Fische schwimmen, wie ein König bzw. eine Prinzessin schreiten und dann auch das Freeze lernen.

In einer ersten Phase des Projekts haben wir uns dabei sehr viel mit den Ideen auseinandergesetzt, die jeder von uns zur Figur Colapesce hatte und die wir mit Gestik, Körperausdruck, Bewegung interpretierten. Es wurden nicht nur die Haltungen der einzelnen Personen interpretiert, sondern auch die einzelnen Elemente der Geschichte, zum Beispiel das Meer, der Sturm, die Fische. Diese grundlegenden Begriffe wurden besser mit dem Spiel der visuellen Interpretationen wahrgenommen, so wie es nachfolgend erläutert wird:

Es ist wichtig, sich dessen bewusst zu sein, dass die Lernenden aufgrund ihrer Vorerfahrungen (und immaginario ancestrale) die Welt und die fremde Sprache wahrnehmen und sich drin ausdrücken. Im Anfängerunterricht kann ein Grundvokabular erworben werden, indem Wörter und Ausdrücke visuell und sinnlich erfahren werden (Tselikas 1999: 70)

In Bezug auf die Darstellungsmethoden haben wir uns von Jerzy Grotowskis (1970) „Theater der Armut“ inspirieren lassen. Er plädiert für ein von allen überflüssigen Requisiten befreites und nur auf den Körper des Schauspielers und seine Kunst beschränktes Theater.

\subsection{Arbeit an der Darstellungs- und Erzählfähigkeit}

Im Projekt wurden die sprachlichen Mittel, die man für das spannende Erzählen in der fremden Sprache benötigt, vorgestellt und auf spielerische Art eingeübt.

- Wir spielten mit Wörtern und ihrem Klang. Die deutsche Sprache eignet sich gut zur Dramatisierung, weil sie so reich an Assonanzen ist, wie die Beispiele aus unserem Text zeigen: Mensch - Fisch - Schiff, Mund - Maul, $\mathrm{Fu} \beta$ - Flosse - Floß.

- Einzelne wurden aufgefordert, spontan auf Fragen der folgenden Art zu reagieren: „Erzähle, warum du ein Fisch geworden bist!“ - „Was reizt dich am königlichen Hof?“ - „Wie fühlst du dich in den Tiefen des Meeres?“

- Verse aus Gedichten von Schiller und Goethe wurden rezitiert, um motivierende Zugänge zum Text über das Experimentieren mit Prosodie entstehen zu lassen. Prosodische Aspekte werden in der Regel an italienischen Schulen und auch andernorts vernachlässigt. ${ }^{2}$

\footnotetext{
${ }^{2}$ Die falsche Aussprache von Einzellauten bei Lernenden nach der Pubertät galt lange Zeit als naturgegeben und unkorrigierbar, die Satzmelodie ist den wenigsten Lehrpersonen selbst vertraut und alle ästhetischen Gesichtspunkte, die beispielsweise bei der Rezitation von Gedichten eine Rolle spielen, waren und sind ohnehin von vornherein aus dem Sprach-Curriculum ausgeklammert und werden ausschließlich dem Literaturunterricht zugewiesen. (siehe Drumbl 2004: 131)
} 
Die einzelnen Treffen mit Singen zu beenden erwies sich als für die Lerner wichtig, um die Musikalität der Sprache zu erfahren. Drei Lieder wurden von der Gruppe während des Projekts erfunden bzw. adaptiert und dann in das Theaterstück eingefügt: „Kleiner Niklas Fisch“, „Froh zu sein bedarf es wenig“ und "Tief, tief".

Mit den Teilnehmern unserer Theatergruppe haben wir dann die Ausdrucksmethoden und Erzähltechniken von Profis (z.B. Ascanio Celestini und Mario Perotta) per Video betrachtet, und im Anschluss daran entsprechende Übungen durchgeführt. Der Schwerpunkt lag dabei im flüssigen Erzählen, denn nur so kann ein Erzähler die Aufmerksamkeit der Zuhörer über längere Zeit fesseln. Die Aufmerksamkeit der Schüler wurde dabei auf Wiederholungseffekte gerichtet, auf Pausen, auf das Erzählen wie in einem intimen Brief usw. Inspirierend für uns wurde auch die Arbeit von Marco Baliani. Er hat mit seiner Inszenierung Pinocchio nero (Der schwarze Pinocchio), an der 20 Jugendliche aus den Slums von Nairobi beteiligt waren, die Geschichte von Carlo Collodis Pinocchio in jenem Kontext neu interpretiert, wobei ein einziger Schauspieler die einzelnen Szenen erzählt.

Zum Üben des Erzählens haben wir der deutschen Übersetzung der Legende Colapesce kleine Auszüge entnommen und diese als Impuls für eigene Erfindungen eingesetzt. Jedes Mal wurde dabei eine Episode aus einem neuen, anderen Blickwinkel (Mutter, Niklas, König, Hofschiffer) erzählt.

Im letzten Monat der Projektphase konnten die Schüler selbstständig die Theaterarbeit durchführen, sie übernahmen die Verantwortung für das Funktionieren der ganzen Inszenierung. Jeder Schauspieler war in allen Szenen aktiv, um zum Beispiel das Meer (eine Plastikfolie) auszubreiten, bei einem Echo-Effekt zu helfen oder im Chor zu singen, Bühnenbildteile ab- und aufzubauen usw. In dieser Schlussphase war es wie im biomechanischen Spiel nach Meyerhold (1907), in dem alle Schauspieler gleichzeitig und zuverlässig, wie die Zahnräder einer Maschine, zusammen funktionieren.

\section{Schlussfolgerungen}

In Turin wurde unsere Leistung mit dem Gewinn des Wettbewerbs gekrönt. Die Schüler waren so begeistert über den Erfolg, dass sie mit großem Einsatz auch an einer Video-Adaption des Theaterstücks mitgewirkt haben. Durch den Kurzfilm können die Schüler ihre Familienangehörige, Freunde oder andere Schulklassen auf ein Projekt aufmerksam machen, mit dem sie sich stark identifizieren und auf diese Weise auch auf die deutsche Sprache neugierig machen.

Als die Theatergruppe im Mai 2007 beim Festival Sprachen in Szene in Turin vor einem europäischen Publikum aufgetreten ist, waren die Schüler zum einen Schauspieler und zum anderen Zuschauer, und durch die eigene Theatererfahrung ein bewusste Zuschauer! Der ganze Prozess der dramatischen Gestaltung, vor allem in diesem europäischen Rahmen, war eine nachhaltig motivierende Erfahrung. In Turin haben die Schüler aus Spanien, Bulgarien, 
Frankreich und, zu unserer besonderen Freude, die deutschen Schüler aus dem Albertus-Magnus-Gymnasium aus Köln am Ende der Aufführung ihre Begeisterung offen gezeigt - ein Beispiel dafür, wie wichtig es ist, in unseren Schulen an die Tradition des theatralen Erzählens, Rezitierens und Singens anzuknüpfen und diese aufrecht zu erhalten.

In einigen Bundesländern Deutschlands ist seit den 70er Jahren Darstellendes Spiel als Schulfach eingeführt worden. Für den Bildungsbereich in Italien wäre eine ähnliche Entwicklung ein großer Gewinn, speziell auch die Förderung von Theaterprojekten mit einem Schwerpunkt auf Fremdsprachenerwerb bzw. Mehrsprachigkeit, ganz im Sinne der Vorstellungen von Maria-Antonia De Libero, Bildungskooperatorin des Goethe-Instituts Turin und Referentin für Mit Deutsch auf die Bühne:

Das Theaterspiel sollte als Unterrichtsform per se fester Bestandteil im schulischen Alltag sein. Dort, wo neben Lernstoffvermittlung Spaß nicht zu kurz kommt, werden auch Lerninhalte besser vermittelt. Kreativität, Lernverhalten und Mut zur Selbstdarstellung sind beim Theaterspiel gefragt. [...] Fremdsprachen können den Ausgangspunkt für „Theater in der Schule“ bilden. (De Libero 2006: 4)

\section{Bibliographie}

Baliani, Marco (2005): Pinocchio nero. Diario di un viaggio teatrale. Milano: Rizzoli

Brauneck, Manfred / Schnellin, Gérard (Hrsg.) (2001): Theaterlexikon. Hamburg: Reinbek

Calvino, Italo (1956): Fiabe Italiane (collana I Meridiani 1993). Milano: Mondadori

Calvino, Italo (1956): Italienische Märchen. [Aus dem Ital. von Lisa Rüdiger]. Zürich: Manesse (1993)

De Libero, Maria-Antonia (2006): Theater in der Schule. In: PerVoi Didaktisch-Kulturelle Zeitschrift für DeutschlehrerInnen. Goethe-Institut Italien 1/2006, 4-12

Drumbl, Johann (2004): Die Stimme im Text. In: Foschi, Albert Marina / Hepp, Marianne / Neuland, Eva (Hrsg.) Texte in Sprachforschung und Sprachunterricht. München: Iudicium, 130-141

Grotowski, Jerzy (1970): Per un teatro povero [Aus dem Engl. (1968) Towards a Poor Theatre von Maria Ornella Marotti, mit dem Vorwort von Peter Brook]. Roma: Bulzoni

von Hentig, Harmut (1996): Bildung. München: Beltz

Karlinger, Felix (1993): Italienische Volksmärchen. München: Diederichs Lippert, Elinor (1998): Theater spielen. Themen, Texte, Interpretationen. Bamberg 
Meyerhold, Wsewolod Emiljewitsch (1907): Theatre. History and Techniques (Neuausgabe 1998). Theatre, London: Methuen

Schewe, Manfred (1993): Fremdsprache inszenieren. Zu Fundierung einer dramapädagogischen Lehr- und Lernpraxis. Oldenburg: Didaktisches Zentrum, Carl von Ossietzky-Universität Oldenburg

Smorti, Andrea (1994): Il pensiero narrativo. Costruzione di storie e sviluppo della conoscenza sociale. Psicologia. Firenze: Giunti

Tselikas, Elektra (1999): Dramapädagogik im Sprachunterricht. Zürich: Orell Füssli

Wilde, Oscar (1913): The Critic as Artist in Intentions. London: Methuen \& Co, 93-217

Wilson, Michael (2006): Storytelling and Theatre: Contemporary Storytellers and Their Art. Houndsmill: Macmillian 